Draft VERSION JULY 22, 2018

Preprint typeset using IATEX style emulateapj v. 11/12/01

\title{
MAPPING LARGE-SCALE CO DEPLETION IN A FILAMENTARY INFRARED DARK CLOUD
}

\author{
Audra K. Hernandez \\ Department of Astronomy, University of Florida, Gainesville, FL 32611, USA; \\ audrah@astro.ufl.edu \\ JONATHAN C. TAN \\ Departments of Astronomy \& Physics, University of Florida, Gainesville, FL 32611, USA; \\ jt@astro.ufl.edu \\ PaOla CASElli \\ School of Physics \& Astronomy, The University of Leeds, Leeds, LS2 9Jt, UK; \\ P.Caselli@leeds.ac.uk \\ MiCHAEL J. BUTLER \\ Department of Astronomy, University of Florida, Gainesville, FL 32611, USA; \\ butler85@astro.ufl.edu \\ IZASKUN JimÉNEZ-SERRA \\ Harvard-Smithsonian Center for Astrophysics, 60 Garden St., 02138, Cambridge, MA, USA; \\ ijimenez-serra@cfa.harvard.edu \\ Francesco FONTANI \\ Institut de Radioastronomie Millimétrique, 300 rue de la Piscine, 38406 St. Martin d'Heres, France; \\ fontani@iram.fr \\ Peter BARNes \\ Department of Astronomy, University of Florida, Gainesville, FL 32611, USA; \\ peterb@astro.ufl.edu \\ Draft version July 22, 2018
}

\begin{abstract}
Infrared Dark Clouds (IRDCs) are cold, high mass surface density and high density structures, likely to be representative of the initial conditions for massive star and star cluster formation. CO emission from IRDCs has the potential to be useful for tracing their dynamics, but may be affected by depleted gas phase abundances due to freeze-out onto dust grains. Here we analyze $\mathrm{C}^{18} \mathrm{O} J=1 \rightarrow 0$ and $J=2 \rightarrow 1$ emission line data, taken with the IRAM $30 \mathrm{~m}$ telescope, of the highly filamentary IRDC G035.39.0033. We derive the excitation temperature as a function of position and velocity, with typical values of $\sim 7 \mathrm{~K}$, and thus derive total mass surface densities, $\Sigma_{\mathrm{C} 18 \mathrm{O}}$, assuming standard gas phase abundances and accounting for optical depth in the line, which can reach values of $\sim 1$. The mass surface densities reach values of $\sim 0.07 \mathrm{~g} \mathrm{~cm}^{-2}$. We compare these results to the mass surface densities derived from mid-infrared (MIR) extinction mapping, $\Sigma_{\mathrm{SMF}}$, by Butler \& Tan, which are expected to be insensitive to the dust temperatures in the cloud. With a significance of $\gtrsim 10 \sigma$, we find $\Sigma_{\mathrm{C} 18 \mathrm{O}} / \Sigma_{\mathrm{SMF}}$ decreases by about a factor of 5 as $\Sigma$ increases from $\sim 0.02$ to $\sim 0.2 \mathrm{~g} \mathrm{~cm}^{-2}$, which we interpret as evidence for CO depletion. Several hundred solar masses are being affected, making this one of the most massive clouds in which $\mathrm{CO}$ depletion has been observed directly. We present a map of the depletion factor in the filament and discuss implications for the formation of the IRDC.
\end{abstract}

Subject headings: ISM: clouds, dust, extinction — stars: formation

\section{INTRODUCTION}

Silhouetted against the Galactic background, Infrared Dark Clouds (IRDCs) are opaque at wavelengths $\sim 10 \mu \mathrm{m}$ (Pérault et al. 1996; Egan et al. 1998), cold ( $T<20 \mathrm{~K}$; Carey et al. 1998; Pillai et al. 2006), and dense $\left(\mathrm{n}_{\mathrm{H}} \geq 10^{3}-10^{5} \mathrm{~cm}^{3}\right.$; Teyssier et al. 2002; Rathborne et al. 2006; Butler \& Tan 2009, hereafter BT09; Peretto \& Fuller 2010). They are likely to be the precursors of massive stars and star clusters as they have similar physical conditions, such as mass surface densities, as regions with such star formation activity (Rathborne et al. 2006; Tan 2007; Zhang et al. 2009; Ragan et al. 2009). CO emission from these clouds may be useful for understanding their dynamics (e.g. Hernandez \& Tan 2011, hereafter HT11), but could be affected by depleted gas phase abundances due to freeze-out onto dust grains, especially in the coldest, highest density regions.

Gas phase depletion of $\mathrm{CO}$, averaged along the line of sight, has been observed in the cold $(T \lesssim 10 \mathrm{~K})$ centers of relatively low-mass and nearby starless cores, (e.g. Willacy et al. 1998; Caselli et al. 1999; Kramer et al. 1999; Bergin et al. 2002; Whittet et al. 2010; Ford \& Shirley 2011). Typically, depletion is characterized by measuring the depletion factor, $f_{D}$, defined as the ratio of $\mathrm{CO}$ column density expected assuming standard gas phase abundances given the column of material observed from either the mm dust continuum emission or near infrared (NIR) dust extinction to the observed $\mathrm{CO}$ column density (typically from $\mathrm{C}^{17} \mathrm{O}$ or $\mathrm{C}^{18} \mathrm{O}$ ). Caselli et al. (1999) estimated the expected $\mathrm{CO}$ column based on $\mathrm{mm}$ dust continuum emission, which has the advantage of being able to probe to high column densities, but is sensitive to the adopted dust temperature and emissivity. They concluded depletion affected a region at the core center containing about 
$2 M_{\odot}$ of gas, where $n_{\mathrm{H}} \gtrsim 10^{5} \mathrm{~cm}^{-3}$, with depletion factors of up to $\sim 10$ where the mass surface density is $\Sigma \simeq 0.6 \mathrm{~g} \mathrm{~cm}{ }^{-2}$. Kramer et al. (1999) estimated the expected CO column based on NIR extinction, which does not require knowing the dust temperature, but does require there to be a sufficient areal density of background stars detectable in the NIR. They found depletion factors of up to $\sim 2.5$ for regions with $A_{V} \sim 20-30 \mathrm{mag}$, corresponding to $\Sigma \sim 0.1-0.15 \mathrm{~g} \mathrm{~cm}^{-2}$.

Massive protostellar cores and clumps are typically more distant and difficult to study, but CO depletion has been reported by Fontani et al. (2006) from a study of 10 sources with median $f_{D} \simeq 3.2$ (but a dispersion of about a factor of 10), Thomas \& Fuller (2008) from a study of 10 sources with a mean $f_{D} \simeq 1.3$ and Lo et al. (2011) from a study of 1 source with $f_{D} \sim 10$. These results rely on estimates of the expected $\mathrm{CO}$ column density based on mm dust continuum emission, are derived only for single pointings to the sources, and can depend on radiative transfer modeling of the unresolved source density and temperature structure (Thomas \& Fuller 2008; Lo et al. 2011). Source to source comparisons are hampered by possible isotopic abundance variations affecting these rare CO isotopologues. The above sources already contain massive protostars, but it is not clear if the depletion signal arises from the immediate surrounding envelope or from nearby unresolved starless cores. Some of the massive protostars studied produce ultra-compact H II regions and photodissociation of molecules could be occurring in localized regions, which would mimic depletion.

We expect CO depletion to be widespread in the dense regions of IRDCs, potentially affecting: the physical properties one derives from $\mathrm{CO}$ emission; the mid and far infrared opacities of dust grains as $\mathrm{CO}$ ice mantles build up; and thus the initial conditions of star and planet formation in these regions. Individual resolved IRDCs, assumed to have uniform isotopic abundances, may also be useful laboratories in which to study the depletion process as a function of local gas conditions.

In this paper, we present IRAM 30m observations of $\mathrm{C}^{18} \mathrm{O} J=1 \rightarrow 0$ and $J=2 \rightarrow 1$ emission from the filamentary IRDC G035.30-00.33 (Cloud H in BT09; near kinematic distance of $d=2.9 \mathrm{kpc}$ ). To look for evidence of depletion, the $\mathrm{C}^{18} \mathrm{O}$-derived mass surface density, $\Sigma_{\mathrm{C} 18 \mathrm{O}}$, is compared with the small median filter (SMF) mid-infrared (MIR) extinction mapping derived mass surface density, $\Sigma_{\mathrm{SMF}}$ (BT09; Butler \& Tan 2011, hereafter BT11). This work is motivated by the study of HT11, who used ${ }^{13} \mathrm{CO}$ molecular line emission from the Galactic Ring Survey (GRS) to estimate the mass surface densities of two highly filamentary IRDCs, including Filament H. Assuming a constant value of $T_{\text {ex }}=15 \mathrm{~K}, \mathrm{HT} 11$ found tentative evidence for $\mathrm{CO}$ depletion, but could not exclude the possibility that other effects, such as systematic changes in the excitation temperature or the contribution of high opacity cores, were the cause of the observed decrease of $\Sigma_{13 \mathrm{CO}} / \Sigma_{\mathrm{SMF}}$ with increasing $\Sigma$. With our new higher-resolution, multi-transition $\mathrm{C}^{18} \mathrm{O}$ data, we are able to exclude or mitigate these effects, as well as resolving higher mass surface density structures to probe a larger range of conditions where depletion may be occurring.

\section{MASS SURFACE DENSITY FROM MIR EXTINCTION MAPPING}

The $8 \mu \mathrm{m}$ SMF mass surface density, $\Sigma_{\mathrm{SMF}}$, map was derived at $2^{\prime \prime}$ resolution from the Spitzer IRAC band 4 (Galactic Legacy Mid-Plane Survey Extraordinaire [GLIMPSE]; Benjamin et al. 2003) image by comparing the observed intensity at each position with the expected background intensity, estimated by interpolating the intensities of surrounding nearby regions where median filter smoothing is used to define the background model (see Figure 1a and 1b). Following BT09, a dust opacity of $\kappa_{8 \mu \mathrm{m}}=7.5 \mathrm{~cm}^{2} \mathrm{~g}^{-1}$ was adopted, similar to the filter response and background spectrum weighted mean IRAC band 4 opacity expected from the Ossenkopf \& Henning (1994) thin ice mantle moderately coagulated grain model with a gas-to-dust mass ratio of 156. This value is somewhat higher than values adopted by other dust models (e.g. 125 is used for the Weingartner \& Draine 2001), although a recent estimate from depletion studies finds a gas-to-dust ratio of 141 (Draine 2011, p265). In any case, as described below, our study of CO depletion compares relative abundances as a function of $\Sigma$ in the IRDC and so is independent of this choice of overall normalization.

A correction for foreground emission also needs to be estimated. BT09 made this correction by estimating the amount of foreground emission from a physical model of the Milky Way and given a measured kinematic distance (assumed to be near) of the cloud. Battersby et al. (2010) have pointed out an additional source of foreground from scattering in the IRAC array. BT11 have developed a more accurate empirical method for estimating the foreground emission, based on the presence of independent saturated (high optical depth) cores, and here we use this new method. For the region we analyze in this particular IRDC, the values of $\Sigma_{\mathrm{SMF}}$ are increased by about $10 \%$ from those presented by BT09.

$\Sigma_{\mathrm{SMF}}$ in the filament is derived from comparison with adjacent regions, which are assumed to have negligible MIR extinction. In reality, we know from molecular line observations (e.g. ${ }^{13} \mathrm{CO}$ from the GRS analyzed by HT11), that these regions do have some material present associated with the IRDC. We refer to this as the IRDC "envelope". The presence of the envelope and other systematic uncertainties associated with estimation of the MIR background intensity mean that $\Sigma_{\mathrm{SMF}}$ becomes unreliable when $\lesssim 0.01 \mathrm{~g} \mathrm{~cm}^{-2}$. For our comparison with the mass surface density derived from $\mathrm{C}^{18} \mathrm{O}$ emission, the $\Sigma_{\mathrm{SMF}}$ map is regridded to the much lower resolution of the $\mathrm{CO}$ data (see below) and all pixels with $\Sigma_{\mathrm{SMF}}<0.01 \mathrm{~g} \mathrm{~cm}^{-2}$ are excluded from the analysis. Methods of accounting for the envelope material are discussed further in 4 .

As noted by BT09, we must also account for locations of bright MIR emission. Wherever the observed MIR intensity is greater than the adopted background model an unphysical negative value of $\Sigma$ will be estimated. Negative values of $\Sigma$ are allowed up to levels comparable with the observed noise, but more extreme values, which are mostly due to discrete MIR bright sources, have $\Sigma_{\text {SMF }}$ set to zero. This causes an underestimation of the mass surface density in these regions. We identify and exclude from further analysis remaining (i.e. $\geq 0.01 \mathrm{~g} \mathrm{~cm}^{-2}$ ) pixels in $\Sigma_{\text {SMF }}$ map (smoothed to the CO resolution) that have more than $20 \%$ of their area occupied by zero or negative values. In Figure $1 \mathrm{~b}$, these excluded 
pixels are indicated with "X" and "O" symbols for the $\mathrm{CO}(1-0)$ and $\mathrm{CO}(2-1)$ resolutions, respectively. Their exclusion is due either to the presence of a MIR bright source or in regions where the background modeling is inaccurate, which can sometimes occur near the edge of the filament. Only a relatively small number of pixels are affected by this exclusion. In fact, our final results would not have varied significantly if this exclusion had not been implemented.

\section{MASS SURFACE DENSITY FROM $\mathrm{C}^{18} \mathrm{O}$ EMISSION}

\subsection{Observations}

The $\mathrm{C}^{18} \mathrm{O} J=1 \rightarrow 0$ and $J=2 \rightarrow 1$ lines were mapped using the IRAM (Instituto de Radioastronomia Milimetrica) $30 \mathrm{~m}$ antenna in Pico Veleta, Spain in August and December 2008. An area of $2^{\prime} \times 4^{\prime}$ was mapped using the On-TheFly (OTF) method towards G035.39-00.33 with a central position of $\alpha(J 2000)=18^{h} 57^{m} 08^{s}, \delta(J 2000)=02^{\circ} 10^{\prime} 30^{\prime \prime}$ $\left(l=35.517^{\circ}, b=-0.274^{\circ}\right)$. While the $\mathrm{C}^{18} \mathrm{O} J=1 \rightarrow 0$ transition was observed with the ABCD receivers with typical single side band (SSB) rejections $>10 \mathrm{~dB}$, the $\mathrm{C}^{18} \mathrm{O} J=2 \rightarrow 1$ lines emission was mapped by using the HERA multibeam receiver. Off-positions for both transition lines were set to $\left(1830^{\prime \prime}, 658^{\prime \prime}\right)$. The beam size at $\sim 110 \mathrm{GHz}$ for the $J=1 \rightarrow 0$ transitions is $22^{\prime \prime}$, while at $\sim 220 \mathrm{GHz}$ the $J=2 \rightarrow 1$ beam size is $11^{\prime \prime}$. The VESPA spectrometer provided spectral resolutions of $20 \mathrm{kHz}$ and $80 \mathrm{kHz}$ for the $J=1 \rightarrow 0$ and $J=2 \rightarrow 1$ lines respectively, which correspond to velocity resolutions of $\sim 0.05 \mathrm{~km} \mathrm{~s}^{-1}$ and $\sim 0.1 \mathrm{~km} \mathrm{~s}^{-1}$. For this study, all spectra were resampled to the same velocity resolution of $0.2 \mathrm{~km} \mathrm{~s}^{-1}$. The typical system temperatures were $150-220 \mathrm{~K}$. Intensities were calibrated in units of antenna temperature $\left(\mathrm{T}_{\mathrm{A}}^{*}\right)$, and converted into a main beam brightness temperature, $\mathrm{T}_{\mathrm{B}, \nu}$, via $T_{A} \equiv \eta f_{\text {clump }} T_{B, \nu}$, where $\eta$ is a main beam efficiency and $f_{\text {clump }}$ is the beam dilution factor. We use $\eta=0.64$ for the $J=1 \rightarrow 0$ transition, and $\eta=0.52$ for the $J=2 \rightarrow 1$ transition. The typical $1 \sigma$ RMS noise of the data is $0.2 K \mathrm{~km} \mathrm{~s}^{-1}$ over the velocity range of $40-50 \mathrm{~km} \mathrm{~s}^{-1}$. Since the $\mathrm{C}^{18} \mathrm{O}$ emission is extended over the filament, we assume $f_{\text {clump }}=1$. Figure 1 p presents the morphology of Filament $\mathrm{H}$ as seen in $\mathrm{C}^{18} \mathrm{O} J=2 \rightarrow 1$ emission.

\subsection{Mass Surface Density and $T_{\mathrm{ex}}$ Estimates}

We estimate the column density of $\mathrm{C}^{18} \mathrm{O}$ molecules, $\mathrm{d} N_{\mathrm{C} 18 \mathrm{O}}$, in the velocity interval $\mathrm{d} v$, from their emission through the general equation:

$$
\frac{\mathrm{d} N_{\mathrm{C} 18 \mathrm{O}}(v)}{\mathrm{d} v}=\frac{8 \pi}{A \lambda_{0}^{3}} \frac{g_{l}}{g_{u}} \frac{\tau_{\nu}}{1-\exp \left(-h \nu / k T_{\mathrm{ex}}\right)} \frac{Q_{\mathrm{rot}}}{g_{l} \exp \left(-E_{l} / k T_{\mathrm{ex}}\right)} .
$$

Here $Q_{\text {rot }}$ is the partition function for linear molecules given by $Q_{\text {rot }}=\sum_{J=0}^{\infty}(2 J+1) \exp \left(-E_{J} / k T_{\text {ex }}\right)$ with $E_{J}=J(J+$ 1) $h B$, where $\mathrm{J}$ is the rotational quantum number and $\mathrm{B}$ is the $\mathrm{C}^{18} \mathrm{O}$ rotational constant equal to $5.4891 \times 10^{10} \mathrm{~s}^{-1}$. $h \nu / k=5.269,10.54 \mathrm{~K}$ for $J=1 \rightarrow 0$ and $J=2 \rightarrow 1$ transitions, respectively. At $7.5 \mathrm{~K}, Q_{\text {rot }}=3.205$. $A$ is the Einstein coefficient, $6.266,60.11 \times 10^{-8} \mathrm{~s}^{-1}$ for $J=1 \rightarrow 0$ and $J=2 \rightarrow 1$, respectively. $\lambda_{0}$ is the wavelength of the transition, $0.273,0.137 \mathrm{~cm}$ for $J=1 \rightarrow 0$ and $J=2 \rightarrow 1$, respectively. $g_{l}$ and $g_{u}$ are the statistical weights of the lower and upper levels, and $\tau_{\nu}$ is the optical depth of the line at frequency $\nu$, i.e. at velocity $v$. The excitation temperature, $T_{\text {ex }}$, is assumed to be the same for all rotational levels. Details on the estimation of $T_{\mathrm{ex}}$ are given below.

The optical depth, $\tau_{\nu}$, is derived through the detection equation:

$$
T_{B, \nu}=\frac{h \nu}{k}\left[f\left(T_{\mathrm{ex}}\right)-f\left(T_{\mathrm{bg}}\right)\right]\left[1-e^{-\tau_{\nu}}\right]
$$

where $T_{B, \nu}$ is the main beam brightness temperature at frequency $\nu, f(T) \equiv[\exp (h \nu /[k T])-1]^{-1}$, and $T_{\mathrm{bg}}$ is the background temperature of $2.725 \mathrm{~K}$. For the observable, $T_{B, \nu}$, and for an assumed $T_{\mathrm{ex}}, \tau_{\nu}$ can be solved for directly through equation (2). Therefore, we can solve for the column density per unit velocity, $\mathrm{d} N_{18 \mathrm{CO}} / \mathrm{d} v$, at each $l, b, v$ position.

While care is taken to account for the optical depth in our column density estimates, for reference we also state the case of the optically thin limit of the $\mathrm{C}^{18} \mathrm{O}(J=1 \rightarrow 0)$ column density. If $\tau_{\nu}$ is small, then equation (2) reduces to $T_{B, \nu}=(h \nu / k)\left[f\left(T_{\mathrm{ex}}\right)-f\left(T_{\mathrm{bg}}\right)\right] \tau_{\nu}$. Inserting into equation (11) gives:

$$
\begin{aligned}
\frac{\mathrm{d} N_{\mathrm{C} 18 \mathrm{O}}(v)}{\mathrm{d} v} & =6.571 \times 10^{14} \frac{Q_{\mathrm{rot}}}{f\left(T_{\mathrm{ex}}\right)-f\left(T_{\mathrm{bg}}\right)}\left[1-\exp \left(-h \nu / k T_{\mathrm{ex}}\right)\right]^{-1} \frac{T_{A} / K}{\eta f_{\text {clump }}} \mathrm{cm}^{-2} \mathrm{~km}^{-1} \mathrm{~s} \\
& \rightarrow 9.758 \times 10^{14} \frac{T_{A}^{*} / K}{\eta f_{\text {clump }}} \mathrm{cm}^{-2} \mathrm{~km}^{-1} \mathrm{~s}\left(T_{\mathrm{ex}}=7.5 \mathrm{~K}\right) .
\end{aligned}
$$

As in HT11, an inspection of the $\mathrm{C}^{18} \mathrm{O}$ emission in $l, b, v$ space indicates that the gas associated with the filament is in the range of $40-50 \mathrm{~km} \mathrm{~s}^{-1}$. The total column density per pixel is then calculated over the entire velocity range of the filament, $N_{\mathrm{C} 18 \mathrm{O}}=\int d N_{\mathrm{C} 18 \mathrm{O}}$.

The column densities for both transitions, $N_{\mathrm{C} 18 \mathrm{O}}$, are converted to a total mass surface density $\Sigma_{\mathrm{C} 18 \mathrm{O}}$, by assuming the abundance ratios of $\mathrm{n}_{16 \mathrm{O}} / \mathrm{n}_{18 \mathrm{O}}=327$ from Wilson \& Rood (1994) and $\mathrm{n}_{12 \mathrm{CO}} / \mathrm{n}_{\mathrm{H} 2}=2 \times 10^{-4}$ from Lacy et al. (1994). Thus, our assumed abundance ratio of $\mathrm{C}^{18} \mathrm{O}$ to $\mathrm{H}_{2}$ is $6.12 \times 10^{-7}$ and $\Sigma$ for each pixel is then given by:

$$
\Sigma_{\mathrm{C} 18 \mathrm{O}}=7.652 \times 10^{-2} \frac{N_{\mathrm{C} 18 \mathrm{O}}}{10^{16} \mathrm{~cm}^{-2}} \mathrm{~g} \mathrm{~cm}^{-2},
$$

assuming a mass per $\mathrm{H}$ nucleus of $\mu_{\mathrm{H}}=2.34 \times 10^{-24} \mathrm{~g}$, i.e. $\Sigma=1 \mathrm{~g} \mathrm{~cm}^{-2}$ is equivalent to $N_{\mathrm{H}}=4.27 \times 10^{23} \mathrm{~cm}^{-2}$. 

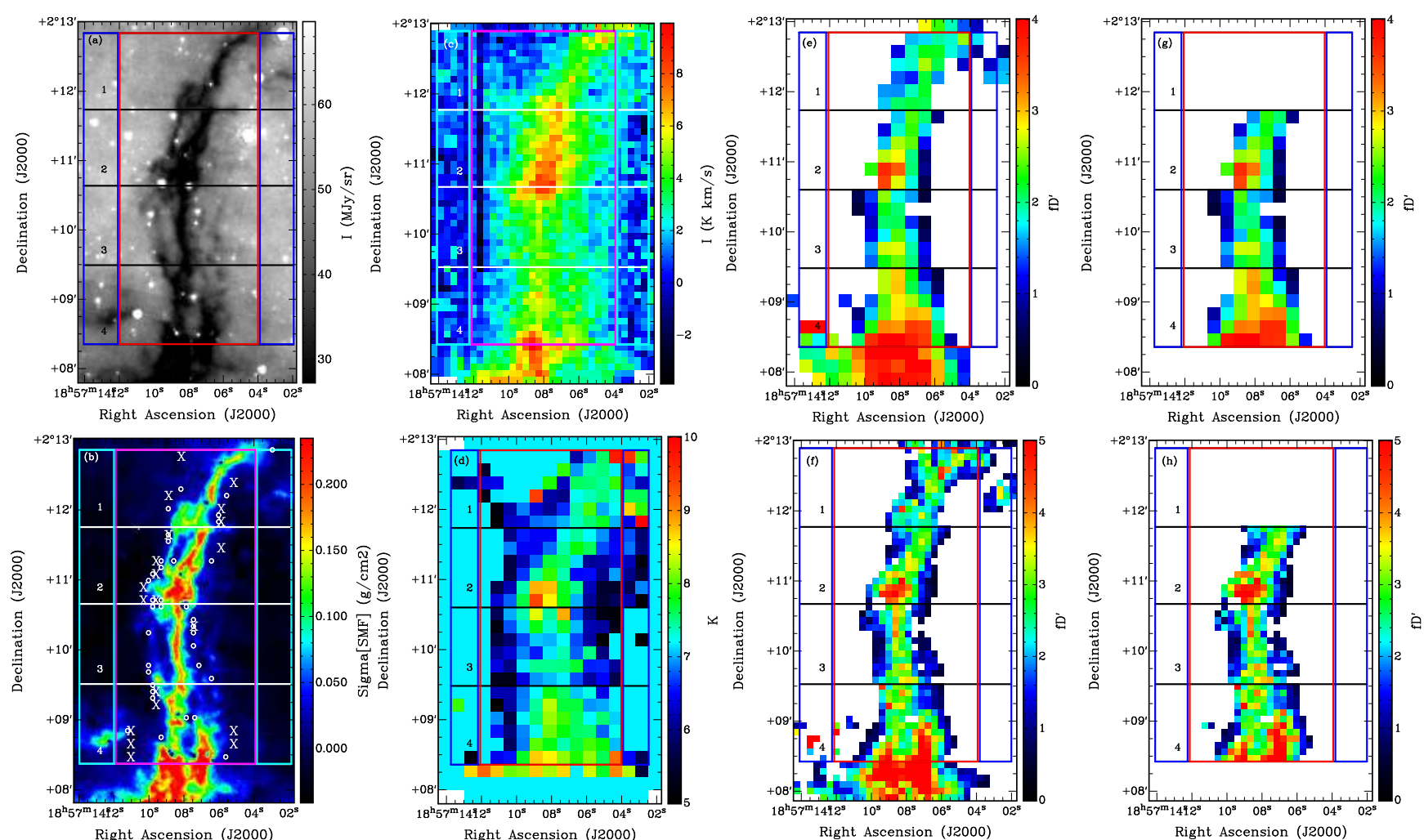

FIG. 1.- Morphology and depletion maps of the IRDC. (a) Top left: Spitzer GLIMPSE IRAC $8 \mu \mathrm{m}$ image, with linear intensity scale in MJy $\mathrm{Sr}^{-1}$. The image has $1.2^{\prime \prime}$ pixels and the PSF has a FWHM of $2^{\prime \prime}$. (b) Bottom left: Mass surface density, $\Sigma_{\mathrm{SMF}}$, with linear intensity scale in $\mathrm{g} \mathrm{cm}^{-2}$, derived from the image in panel (a) using the small median filter (SMF) MIR extinction mapping method of Butler \& Tan (2009; 2011). Regions with $\Sigma_{\mathrm{SMF}}>0.01 \mathrm{~g} \mathrm{~cm}^{-2}$ but which are $>20 \%$ affected by artifacts in the extinction map (e.g. due to MIR bright sources) are excluded from analysis and shown by "X"'s and "O"'s for $\mathrm{CO}(1-0)$ and (2-1) resolution grids, respectively. (c) Top middle left: Integrated intensity map of $\mathrm{C}^{18} \mathrm{O}(J=2 \rightarrow 1)$ emission over the velocity range of $40-50 \mathrm{~km} \mathrm{~s}^{-1}$, i.e. the gas associated with the IRDC (HT11), in linear units of $\mathrm{K} \mathrm{km} \mathrm{s}^{-1}$ and a pixel scale of $5^{\prime \prime}$. (d) Bottom middle left: The mean excitation temperature map weighted by the column density in $\mathrm{K}$, with pixel size of $11^{\prime \prime}$. ( $(e)$ Top middle right: Relative depletion factor $\left(f_{D}^{\prime}\right)$ map for Case 1 (no CO envelope subtraction). (f) Bottom middle right: Relative depletion factor ( $\left.f_{D}^{\prime}\right)$ map for Case 1 HiRes (no $\mathrm{CO}$ envelope subtraction, $\Sigma$ derived at the $\mathrm{CO}(2-1)$ resolution). (g) Top right: Relative depletion factor map for Case 2 (CO envelope contribution estimated via interpolation across strips 2, 3 and 4 then subtracted; note we consider this process unreliable for strip 1). (h) Bottom right: Relative depletion factor map for Case 2 HiRes. 
In order to accurately derive the mass surface density of the filament, an estimate of the excitation temperature, $T_{\text {ex }}$, is needed. To perform this estimate throughout the filament, we varied the assumed temperature at each $l, b, v$ position until the ratio between the column densities derived from both transitions were in agreement. To do this, we first defined $R_{2,1}$ as the ratio between the $J=2 \rightarrow 1$ and $J=1 \rightarrow 0$ column densities:

$$
R_{2,1} \equiv \frac{\mathrm{d} N_{\mathrm{C} 18 \mathrm{O}, 21}}{\mathrm{~d} N_{\mathrm{C} 18 \mathrm{O}, 10}} .
$$

This method is similar to the one used in Kramer et al. (1999), except they averaged over the velocity profile of their cloud. The higher resolution $J=2 \rightarrow 1$ data was convolved with a beam of $22^{\prime \prime}$ and regridded to match the resolution and pixel scale of the $J=1 \rightarrow 0$ data. For all $l, b, v$ positions above a noise limit of $3 \sigma$ in both transitions, $R_{2,1}$ was calculated first assuming a $T_{\mathrm{ex}}=30 \mathrm{~K}$. Then, $T_{\mathrm{ex}}$ was iteratively decreased until $R_{2,1}$ converged to unity. This step provided a three dimensional grid containing estimates of $T_{\text {ex }}$ for all positions above the noise limit. Next, for all positions below the noise threshold, their $T_{\text {ex }}$ was estimated by taking the mean excitation temperature at the corresponding $l, b$ position. Finally, for any remaining $l, b, v$ positions without an estimated excitation temperature, the mean $T_{\text {ex }}$ of $7.2 \mathrm{~K}$ resulting from the previous steps was used. Positions left for this final step are mainly in the outer regions of the filament where the emission is weak and/or the noise is high. The column density weighted $T_{\mathrm{ex}}$ map is shown in Figure 1d.

\section{COMPARISON OF $\Sigma_{\mathrm{C} 18 \mathrm{O}}$ AND $\Sigma_{\mathrm{SMF}}$ : EVIDENCE FOR CO DEPLETION}

In Figure 1, we present the morphology of the filamentary IRDC H. The goal of this section is to compare $\Sigma_{\mathrm{C} 18 \mathrm{O}}$ and $\Sigma_{\mathrm{SMF}}$. The simplest way of doing this, which we refer to as Case 1, involves a straightforward pixel by pixel comparison of these values, smoothing the $\Sigma_{\mathrm{SMF}}$ data to the resolution of the $\mathrm{C}^{18} \mathrm{O}(1-0)$ observations, for which we have derived accurate excitation temperature information. Note, that only pixels with $\Sigma_{\mathrm{SMF}}$ and $\Sigma_{\mathrm{C} 18 \mathrm{O}} \geq 0.01 \mathrm{~g} \mathrm{~cm}^{-2}$ are considered. Also, pixels for which $\Sigma_{\mathrm{SMF}}$ is affected by bright MIR emission are excluded (see 乌2). We also perform a comparison at the higher angular resolution of the $\mathrm{C}^{18} \mathrm{O}(2-1)$ observations, which we refer to as Case 1 HiRes, assuming $T_{\text {ex }}$ at this higher angular resolution can be estimated from the values derived at the (1-0) resolution. For both these versions of Case 1, we refer to $\Sigma_{\mathrm{C} 18 \mathrm{O}}$ as $\Sigma_{\mathrm{C} 18 \mathrm{O} \text {,TOT }}$, since it is derived from all the $\mathrm{C}^{18} \mathrm{O}$ emission associated with the IRDC and its surrounding GMC.

However, as is apparent from Figure 1, the $\mathrm{C}^{18} \mathrm{O}$ emission is more extended than the $8 \mu$ m extinction map from Butler \& Tan $(2009 ; 2011)$. This is because, as discussed above, the extinction map is derived from an "on-off" comparison with adjacent regions, which help define the background MIR intensity that is expected to be behind the filament. Thus the MIR extinction mapping method becomes insensitive to material present in these adjacent, lower column density ("envelope") regions. A fair comparison between $\Sigma_{\mathrm{SMF}}$ and $\Sigma_{\mathrm{C} 18 \mathrm{O}}$ would allow for this envelope material. We thus define "filament" and "envelope" regions based on the $8 \mu \mathrm{m}$ image of the IRDC. Following HT11, the filament is defined to be a rectangular strip centered at $\alpha(J 2000)=18^{h} 57^{m} 08.02^{s}, \delta(J 2000)=02^{\circ} 10^{\prime} 35.7^{\prime \prime}, 2.05^{\prime}$ wide in R.A. and $4.47^{\prime}$ long in Dec. The outline of this filament region is shown by a red box in the panels of Figure 1 . The envelope region is defined to be made up of two adjacent rectangular regions on either side of the filament. These are shown as blue rectangles in Figure 1 and are each $0.56^{\prime}$ wide in R.A. and $4.47^{\prime}$ long in Dec. Note, that because of the limited area mapped by our observations, these envelope regions are narrower than those considered by HT11.

For our Case 2, we assume that the $\mathrm{C}^{18} \mathrm{O}$ material present in the envelope regions is also present at the similar levels towards the filament region, and so attempt to subtract this emission from the $\mathrm{C}^{18} \mathrm{O}$ spectrum of the filament, before then comparing to $\Sigma_{\mathrm{SMF}}$. To carry out this subtraction we divide the filament and envelope into four E-W strips (1 to 4 from $\mathrm{N}$ to $\mathrm{S}$ ) (see Figure 1). In each strip, the mean column density per unit velocity is evaluated for the filament (based on $66 \mathrm{C}^{18} \mathrm{O}(1-0)$ pixels) and the two adjacent envelope regions (based on $18 \mathrm{C}^{18} \mathrm{O}(1-0)$ pixels each) (see Figure 2), using the $T_{\text {ex }}$ estimates described previously. The envelope spectra are averaged and then subtracted from the filament. The total column of this envelope-subtracted spectrum is evaluated and used to derive $\Sigma_{\mathrm{C} 18 \mathrm{O} \text {,FIL }}$. This is of course an approximate method for accounting for the envelope material: one can see from Figure 2 that the envelope spectra on either side of the filament can be quite different, especially for strips 1 and 2. The uncertainty in the envelope-subtracted spectrum becomes large when the envelope spectra are of similar strength as that of the filament, as is the case for strip 1 . Thus we do not regard the results of envelope subtraction for strip 1 as being reliable, and we exclude these pixels from the Case 2 analysis. As with Case 1, we also perform a Case 2 HiRes analysis, using $\Sigma_{\mathrm{C} 18 \mathrm{O} \text {,FIL }}$ estimated at the higher resolution of the $\mathrm{CO}(2-1)$ data, adopting values of $T_{\mathrm{ex}}$ evaluated at the $\mathrm{CO}(1-0)$ resolution.

With these Case 1 and 2 methods, we now compare the pixel by pixel values of $\Sigma_{\mathrm{C} 18 \mathrm{O}}$ with $\Sigma_{\mathrm{SMF}}$ derived from MIR extinction mapping. As noted in HT11, these measurements of $\Sigma$ are essentially independent of cloud distance

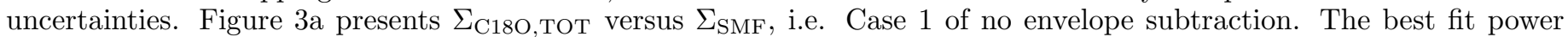
law relation to the $\mathrm{CO}(1-0)$ resolution data of $\Sigma_{\mathrm{C} 18 \mathrm{O} \text {, ТОт }} / \mathrm{g} \mathrm{cm}^{-2}=A\left(\Sigma_{\mathrm{SMF}} / \mathrm{g} \mathrm{cm}^{-2}\right)^{\alpha}$ has $\alpha=0.452 \pm 0.054$ and $A=0.146 \pm 0.023$. For Case 1 HiRes (i.e. at the $\mathrm{CO}(2-1)$ resolution, adopting $\mathrm{CO}(1-0)$ resolution $T_{\text {ex }}$ estimates) we find $\alpha=0.463 \pm 0.025$ and $A=0.151 \pm 0.010$. These results are summarized in Table 1 .

These uncertainties are derived assuming that the errors of each individual measurement are as follows: for $\Sigma_{\mathrm{C} 18 \mathrm{O}}$, a fixed value of $0.0024 \mathrm{~g} \mathrm{~cm}^{-2}$ (derived from the $1 \sigma$ RMS noise of $0.2 \mathrm{~K} \mathrm{~km} \mathrm{~s}^{-1}$ over the velocity range of $40-50 \mathrm{~km} \mathrm{~s}-1$ ) and a $20 \%$ error to account for uncertainties in $T_{\text {ex }}$ assumed to be $1 \mathrm{~K}$ at the typical temperature of $7 \mathrm{~K}$; for $\Sigma_{\mathrm{SMF}}$, a $15 \%$ error plus a systematic error of $0.01 \mathrm{~g} \mathrm{~cm}^{-2}$ (BT09). At the resolution of the CO pixels $\left(11^{\prime \prime}\right.$ for $\mathrm{CO}(1-0)$ and $5^{\prime \prime}$ for 
$\mathrm{CO}(2-1))$, the $\Sigma_{\mathrm{SMF}}$ measurements are independent, but the $\Sigma_{\mathrm{C} 18 \mathrm{O}}$ results are not since the telescope beam is about twice the pixel scale. Thus the above quoted uncertainties of the power law fits assume, conservatively, only $25 \%$ of the pixels are used (although the derived values of the parameters are based on fits to all of the pixels).

We argue below that $\Sigma_{\mathrm{SMF}}$ is a more accurate measure of the true mass surface density in IRDCs than $\Sigma_{\mathrm{C} 18 \mathrm{O}}$, since one does not expect large changes in MIR dust opacities in these environments, based on the Ossenkopf \& Henning (1994) dust models. If this is true, then if $\mathrm{C}^{18} \mathrm{O}$ were also an accurate tracer of mass surface density, then we should see a one-to-one relation between $\Sigma_{\mathrm{C} 18 \mathrm{O}}$ and $\Sigma_{\mathrm{SMF}}$, i.e. $\alpha \simeq 1$, even if $A$ (the value of $\Sigma_{\mathrm{C} 18 \mathrm{O}} / \Sigma_{\mathrm{SMF}}$ when $\Sigma_{\mathrm{SMF}}=1 \mathrm{~g} \mathrm{~cm}^{-2}$ ) is not exactly unity because of systematic uncertainties in the absolute values of $\mathrm{C}^{18} \mathrm{O}$ abundance or MIR dust opacities. We measure $\alpha=0.452 \pm 0.054$ for Case 1 and $\alpha=0.463 \pm 0.025$ for Case 1 HiRes, which are significantly $(10 \sigma$ and $21 \sigma)$ different from one, and we interpret these results as being evidence for $\mathrm{CO}$ depletion from the gas phase.

To illustrate that these results do not depend on the choice of dust opacity per unit gas mass, we have repeated the analysis but with a gas-to-dust mass ratio of 100 (rather than our fiducial value of 156). We find $\alpha=0.509 \pm 0.073$ (about $7 \sigma$ different from $\alpha=1$ ) for Case 1 and $\alpha=0.552 \pm 0.035$ (about $13 \sigma$ different from $\alpha=1$ ) for Case 1 HiRes. Note that we do not expect to derive exactly the same values of $\alpha$ as before since we have a fixed threshold of $\Sigma \geq 0.01 \mathrm{~g} \mathrm{~cm}^{-2}$ to include points in the analysis and so reducing the gas-to-dust mass ratio causes us to lose some data points near this limit.

Figure 3b shows the ratio $\Sigma_{\mathrm{C} 18 \mathrm{O} \text {,тОт }} / \Sigma_{\mathrm{SMF}}$ versus $\Sigma_{\mathrm{SMF}}$ for our fiducial Case 1 and Case 1 HiRes analyses, with the derived power law relations overlaid. For $0.01<\Sigma_{\mathrm{SMF}} / \mathrm{g} \mathrm{cm}^{-2}<0.03$ the mean values of $\Sigma_{\mathrm{C} 18 \mathrm{O} \text {, тOT }} / \Sigma_{\mathrm{SMF}}$ are 1.316 and 1.471 for Case 1 and Case 1 HiRes, respectively. By the time $\Sigma_{\mathrm{SMF}} \gtrsim 0.1 \mathrm{~g} \mathrm{~cm}^{-2}, \Sigma_{\mathrm{C} 18 \mathrm{O}, \mathrm{TOT}} / \Sigma_{\mathrm{SMF}}$ has declined to values of $\lesssim 0.4$.

In Case 2 we attempt to account for the IRDC envelope: we consider that we can do this reliably only for strips 2 , 3 and 4 , where the envelope is relatively weak compared to the filament. Figure 3 p presents $\Sigma_{\text {C18O,FIL versus }} \Sigma_{\text {SMF }}$ for Case 2. The best fit power law relation to the $\mathrm{CO}(1-0)$ resolution data of $\Sigma_{\mathrm{C} 18 \mathrm{O} \text {,FIL }} / \mathrm{g} \mathrm{cm}^{-2}=A\left(\Sigma_{\mathrm{SMF}} / \mathrm{g} \mathrm{cm}^{-2}\right)^{\alpha} \mathrm{has}^{-}$ $\alpha=0.239 \pm 0.080$ and $A=0.074 \pm 0.017$. For Case 2 HiRes (i.e. at the $\mathrm{CO}(2-1)$ resolution, adopting $\mathrm{CO}(1-0) T_{\text {ex }}$ estimates) we find $\alpha=0.317 \pm 0.038$ and $A=0.090 \pm 0.010$. These uncertainties assume the same measurement uncertainties as Case 1, except an additional systematic error of $0.01 \mathrm{~g} \mathrm{~cm}^{-2}$ has been applied to $\Sigma_{\mathrm{C} 18 \mathrm{O} \text {,FIL }}$ due to uncertainties associated with envelope subtraction. Again these results indicate a significant ( $10 \sigma$ and $18 \sigma$ for Case 2 and Case 2 HiRes, respectively) departure from a one-to-one $(\alpha=1)$ relation, which we again interpret as evidence for CO depletion. The results with a gas-to-dust mass ratio of 100 are $\alpha=0.303 \pm 0.11$ (about $6 \sigma$ different from $\alpha=1$ ) for Case 2 and $\alpha=0.372 \pm 0.048$ (about $13 \sigma$ different from $\alpha=1$ ) for Case 2 HiRes.

Figure 3d shows the ratio $\Sigma_{\mathrm{C} 18 \mathrm{O}, \mathrm{FIL}} / \Sigma_{\mathrm{SMF}}$ versus $\Sigma_{\mathrm{SMF}}$ for Case 2 and Case 2 HiRes, with the above power law relations overlaid. For $0.01<\Sigma_{\mathrm{SMF}} / \mathrm{g} \mathrm{cm}^{-2}<0.03$ the mean values of $\Sigma_{\mathrm{C} 18 \mathrm{O}, \mathrm{FIL}} / \Sigma_{\mathrm{SMF}}$ are 1.099 and 1.238 for Case 2 and Case 2 HiRes, respectively. These values are smaller than their equivalents for Case 1, as is to be expected now that we are allowing for the molecular envelope. The values are also very close to unity, suggesting that our adopted $\mathrm{C}^{18} \mathrm{O}$ abundances and dust opacity per unit gas mass are reasonable. Again, by the time $\Sigma_{\mathrm{SMF}} \gtrsim 0.1 \mathrm{~g} \mathrm{~cm}^{-2}, \Sigma_{\mathrm{C} 18 \mathrm{O}, \mathrm{TOT}} / \Sigma_{\mathrm{SMF}}$ has declined to values of $\lesssim 0.4$.

\subsection{Alternatives to CO Depletion}

There are several physical processes that could be responsible for the observed trend of decreasing $\Sigma_{\mathrm{C} 18 \mathrm{O}} / \Sigma_{\mathrm{SMF}}$ with increasing $\Sigma_{\mathrm{SMF}}$. One possibility could be that our corrections for the optical depth of the $\mathrm{C}^{18} \mathrm{O}$ emission are systematically underestimated near the center of the filament where the column density is large. However, the largest optical depth corrections in the highest column density locations increase the column by only $30 \%$ (the highest optical depths are $\sim 1$, but lower when averaged over the whole column), so this effect is unlikely to be driving the observed trend.

HT11 suggested their observed trend of decreasing $\Sigma_{13 \mathrm{CO}} / \Sigma_{\mathrm{SMF}}$ with increasing $\Sigma_{\mathrm{SMF}}$ could potentially result if at the same time there is a systematic decrease in the excitation temperature of about $5 \mathrm{~K}$. However, from our $T_{\mathrm{ex}}$ estimates, we find no strong negative temperature gradient within the IRDC towards the mass surface density peaks. In fact, $T_{\mathrm{ex}}$ increases slightly towards to the center of the filament, probably as the densities become greater than the effective critical densities and the lower $\mathrm{CO}$ levels can thermalize. Thus, we exclude trends in $T_{\mathrm{ex}}$ as causing the observed variation of $\Sigma_{\mathrm{C} 18 \mathrm{O}} / \Sigma_{\mathrm{SMF}}$.

Fractionation of $\mathrm{C}^{18} \mathrm{O}$ could in principle change the local abundance of this molecule, but the most important way in which this can be achieved is via isotope selective photodissociation at cloud edges, which would not be able to explain the trends of decreasing $\mathrm{C}^{18} \mathrm{O}$ abundance that we see running from $\Sigma \simeq 0.02 \mathrm{~g} \mathrm{~cm}^{-2}$ ( $\left.A_{V} \simeq 4 \mathrm{mag}\right)$ to $\simeq 0.2 \mathrm{~g} \mathrm{~cm}^{-2}$ $\left(A_{V} \simeq 40 \mathrm{mag}\right)$.

Another possibility to be considered is systematic changes in $8 \mu \mathrm{m}$ dust opacities for gas at higher densities. If the opacity was to increase (e.g. due to grain coagulation and/or ice mantle formation and growth), then this could explain our observed trend of decreasing $\Sigma_{\mathrm{C} 18 \mathrm{O}} / \Sigma_{\mathrm{SMF}}$ with increasing $\Sigma$. The Ossenkopf \& Henning (1994) dust models do show an increase of $\kappa_{8 \mu \mathrm{m}}$ of $19 \%$ going from the uncoagulated thin ice mantle model to the uncoagulated thick ice mantle (all volatiles depleted) model. Maximal coagulation (corresponding to that expected after $10^{5} \mathrm{yr}$ at densities of $10^{8} \mathrm{~cm}^{-3}$ or after $\sim 10^{8} \mathrm{yr}$ at densities of $\sim 10^{5} \mathrm{~cm}^{-3}$, which is probably more that can be expected to have occurred since the observed densities of IRDC cores are $\lesssim 10^{5} \mathrm{~cm}^{-3}$; BT09) raises $\kappa_{8 \mu \mathrm{m}}$ by an additional $17 \%$. Thus, ice mantle growth and grain coagulation appears to be able to account for only a small fraction of the observed variation of $\Sigma_{\mathrm{C} 18 \mathrm{O}} / \Sigma_{\mathrm{SMF}}$.

We conclude the most likely cause of the trend of decreasing $\Sigma_{\mathrm{C} 18 \mathrm{O}} / \Sigma_{\mathrm{SMF}}$ with increasing $\Sigma_{\mathrm{SMF}}$ is CO depletion due 
to freeze out onto dust grains. This would cause a systematic reduction in the amount of CO gas observed in higher mass surface density regions, which are likely to also be of higher volume density.

\subsection{CO Depletion and Implications}

Following the definitions of $\S 1$ and the notation of Fontani et al. (2006), the depletion factor is

$$
f_{D} \equiv \frac{X_{\mathrm{CO}}^{E}}{X_{\mathrm{CO}}^{O}}=\frac{\Sigma_{\mathrm{SMF}}}{\Sigma_{\mathrm{C} 18 \mathrm{O}}},
$$

where $X_{\mathrm{CO}}^{E}$ is the expected abundance of $\mathrm{CO}$ relative to $\mathrm{H}_{2}$ given standard gas phase abundances, $X_{\mathrm{CO}}^{O}$ is the observed abundance and the last equality assumes that $\Sigma_{\mathrm{SMF}}$ estimated from MIR extinction mapping is an accurate measure of the true mass surface density (this assumption is discussed further below). Given the uncertainties in the absolute values of the $\mathrm{C}^{18} \mathrm{O}$ abundance and the MIR dust opacity per unit gas mass, we renormalize $f_{D}$ to be unity for the regions of the IRDC with $0.01<\Sigma_{\mathrm{SMF}} / \mathrm{g} \mathrm{cm}^{-2}<0.03$ and refer to this renormalized value as the relative depletion factor $f_{D}^{\prime}=B f_{D}$, where the scaling factor, $B=1.316,1.471,1.099,1.238$ for Case 1, Case 1 HiRes, Case 2, Case 2 HiRes, respectively. We show maps of $f_{D}^{\prime}$ for these four cases in Figure 1 $\mathrm{k}$-h. We note that the values of $f_{D}^{\prime}$ presented here, peaking at values $\simeq 5$, are mass surface density weighted averages and thus lower limits to the maximum values of the depletion factor that apply in the densest regions of the cloud.

We conclude that with high $(\sim 10 \sigma)$ significance, widespread CO depletion is occurring in this IRDC, with depletion factors of up to $\sim 5$ (see Table1). These values are larger than those seen towards more evolved cores and clumps already containing massive protostars (Fontani et al. 2006; Thomas \& Fuller 2008). Our measurement of CO depletion suffers from fewer systematic uncertainties, especially since we do not require knowledge of the dust temperature.

Each pixel in the lower resolution depletion maps $\left(11^{\prime \prime}\right.$, half the $\mathrm{C}^{18} \mathrm{O}(1-0)$ angular resolution) corresponds to a length of $0.155 \mathrm{pc}$ at the cloud distance of $2.9 \mathrm{kpc}$, and so contains a mass of $11.4\left(\Sigma / 0.1 \mathrm{~g} \mathrm{~cm}^{-2}\right) M_{\odot}$. Thus, hundreds of solar masses appear to be affected by depletion along the filament (the total SMF-derived mass in the 4 strips is $580 \pm 230 M_{\odot}$, HT11), including a particularly prominent massive core or clump in strip 2 and a larger clump partially in strip 4 and extending to the south.

Thus, IRDC G035.30-00.33 is one of the most massive clouds in which CO depletion has been detected by direct CObased and non-CO-based measurements of mass surface density. Our results also suggest that CO depletion will be a common occurrence in IRDCs, since the values of $\Sigma \sim 0.1 \mathrm{~g} \mathrm{~cm}^{-2}$ in this cloud are quite typical (e.g. BT09). CO is therefore an imperfect tracer of a significant fraction of the mass of IRDCs (not just the coldest, densest cores). Accurate accounting for depletion and/or use of species suffering minimal depletion, such as $\mathrm{NH}_{3}$ and $\mathrm{N}_{2} \mathrm{H}^{+}$, are required for more accurate dynamical studies of these clouds.

An estimate of the CO depletion timescale due to freeze-out onto dust grains is $t_{D} \simeq 8000 /\left(n_{\mathrm{H}_{2}, 5} S\right)$ yr, where $n_{\mathrm{H}_{2}, 5}$ is the number density of $\mathrm{H}_{2}$ molecules in units of $10^{5} \mathrm{~cm}^{-3}$ and $S$ is the sticking probability (of order unity; e.g. Tielens \& Allamandola 1987) for CO on grains. We can apply this to the thinnest region of the IRDC: the $\sim 5^{\prime \prime}(0.070 \mathrm{pc})$ wide filament near the center of strip 3, which appears to have significant CO depletion with $f_{D}^{\prime} \sim 3-4$. Assuming the depth of the filament, which has $\Sigma_{\mathrm{SMF}} \simeq 0.2 \mathrm{~g} \mathrm{~cm}^{-2}$, is similar to its width, then $n_{\mathrm{H}_{2}, 5}=2.0$ and $t_{D} \simeq 4000 \mathrm{yr}$. This provides a lower limit to the age of this part of the IRDC. The free-fall time, $t_{\mathrm{ff}}=(3 \pi /[32 G \rho])^{1 / 2}$, for this density is $6.9 \times 10^{4} \mathrm{yr}$, i.e. much longer. However, if the filament has been created by larger scale supersonic flows, then one might expect the high density gas to have been present for about the flow crossing time across the width of the filament. Velocities of $\sim 10 \mathrm{~km} \mathrm{~s}^{-1}$ may be relevant in models of GMC-GMC collisions (Tan 2000) or if the large-scale SiO emission seen towards this filament (Jiménez-Serra et al. 2010) has been created by such flows. The flow crossing time at this speed for this part of the IRDC is only $6800 \mathrm{yr}$. Thus the fact that we see CO depletion in these very thin filaments of the IRDC can help to constrain models for the cloud's formation. For models in which the cloud lifetime is less than the flow crossing time across the filament, a constraint is placed on the flow speed. For the thinnest region of this IRDC, this corresponds to flow speeds $\lesssim 17 \mathrm{~km} \mathrm{~s}^{-1}$.

We thank E. van Dishoeck and R. Visser for helpful discussions and the comments of an anonymous referee, which helped improve the paper. AKH acknowledges support from a SEAGEP Dissertation Fellowship. JCT acknowledges support from NSF CAREER grant AST-0645412; NASA Astrophysics Theory and Fundamental Physics grant ATP090094; NASA Astrophysics Data Analysis Program ADAP10-0110 and a Faculty Enhancement Opportunity grant from the University of Florida.

TABLE 1

Parameters of Depletion Factor Analysis

\begin{tabular}{lcccc}
\hline \hline Case & $\alpha$ & $A$ & $B$ & $f_{D}^{\prime}(\max )$ \\
\hline Case 1 & $0.452 \pm 0.054$ & $0.146 \pm 0.023$ & 1.316 & $3.5\left(\right.$ at $\left.\Sigma_{\text {SMF }}=0.16 \mathrm{~g} \mathrm{~cm}^{-2}\right)$ \\
Case 1 HiRes & $0.463 \pm 0.025$ & $0.151 \pm 0.010$ & 1.471 & $4.6\left(\right.$ at $\left.\Sigma_{\text {SMF }}=0.20 \mathrm{~g} \mathrm{~cm}^{-2}\right)$ \\
Case 2 & $0.239 \pm 0.080$ & $0.074 \pm 0.017$ & 1.099 & $3.8\left(\right.$ at $\left.\Sigma_{\text {SMF }}=0.16 \mathrm{~g} \mathrm{~cm}^{-2}\right)$ \\
Case 2 HiRes & $0.317 \pm 0.038$ & $0.090 \pm 0.010$ & 1.238 & $4.9\left(\right.$ at $\left.\Sigma_{\text {SMF }}=0.20 \mathrm{~g} \mathrm{~cm}^{-2}\right)$ \\
\hline
\end{tabular}



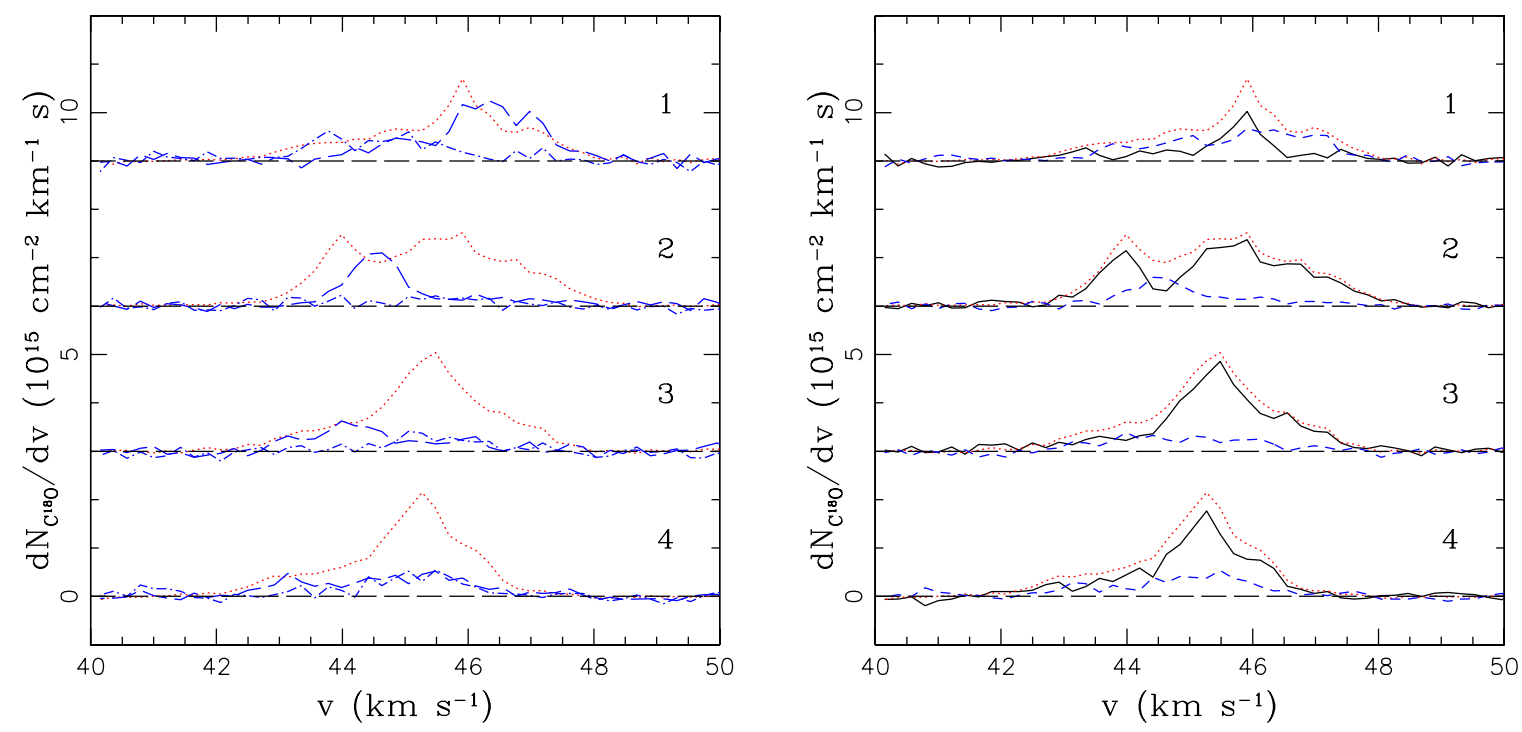

FIG. 2.- Velocity structure of the $\mathrm{C}^{18} \mathrm{O}$ molecules associated with the IRDC and its envelope. The column density distribution, $\mathrm{d} N_{\mathrm{C}^{18} \mathrm{O}} / \mathrm{d} v$, has been derived from the $\mathrm{C}^{18} \mathrm{O}(1-0)$ and (2-1) spectra, local estimates of $T_{\text {ex }}$ and including optical depth corrections. (a) Left: the 4 sets of profiles (offset to display from top to bottom and labeled 1 to 4 ) correspond to the 4 strips shown in Figure 1. The dotted, red line is the summed contribution from gas from the central region of each strip, corresponding to the IRDC "filament" (see Figure 1 and text). The dot-dashed and long-dashed blue lines show summed contribution from the gas from the eastern and western envelope regions, respectively. (b) Right: Illustration of envelope subtraction (Case 2, see text). For the same strips as in (a), we subtract the average of the eastern and western envelopes (short-dashed blue lines) from the filament (dotted red lines), to leave an estimate of the material in the filament (solid black lines). We consider this process unreliable for strip 1, where the envelope contains a similar amount of material as the filament.

\section{REFERENCES}

Battersby, C., Bally, J., Jackson, J. M. et al. 2010, ApJ, 721, 222

Benjamin, R. A., Churchwell, E., Babler, B. L., Bania, T. M., Clemens, D. P. et al. 2003, PASP, 115, 953

Bergin, E. A., Snell, R. L., 2002, ApJ, 581L, 105B

Butler M. J., \& Tan J. C. 2009, ApJ, 696, 484 (BT09)

Butler M. J., \& Tan J. C. 2011, in prep.(BT11)

Brady Ford, A., Shirley, Y.L. 2011, ApJ, 728, 144B

Carey, S. J., Clark, F. O., Egan, M. P., Price, S. D., Shipman, R. F., \& Kuchar, T. A. 1998, ApJ, 508, 721

Carey, S. J., Feldman, P. A., Redman, R. O., Egan, M. P., MacLeod, J. M., \& Price, S. D. 2000, ApJ, 508, 721

Caselli, P., Walmsley, C. M., Tafalla, M., Dore, L., \& Myers, P. C. 1999, ApJ, 523, L165

Draine B. T. 2011, Physics of the interstellar and intergalactic medium, Princeton University Press, Princeton

Egan, M. P., Shipman, R. F., Price, S. D., Carey, S. J., Clark, F. O., \& Cohen, M. 1998, ApJ, 494, L199

Fontani, F., Caselli, P., Crapsi, A., Cesaroni., R., Molinari, S., Testi, L., Brand, J. 2006, A\&A, 460, 790F

Frerking, M. A., Langer, W. D., Wilson, R. W., 1982, ApJ, 262, 590F

Hernandez, A. K., Tan, J. C., 2011, ApJ, accepted, (arXiv1101.1589) (HT11)

Jiménez-Serra, I., Caselli, P., Tan, J. C., Hernandez, A. K., Fontani, F., Butler, M. J., Van Loo, S. 2010, MNRAS, 406, 187

Kramer, C., Alves, J., Lada, C. J., Lada, E. A., Sievers, A., Ungerechts, H., Walmsley, C. M., 1999, A\&A, 342, 257

Lacy, J. H.; Knacke, R.; Geballe, T. R.; Tokunaga, A. T. 1994, ApJ, 428, L69
Lo, N., Redman, M. P., Jones, P. A., Cunningham, M. R., Chhetri, R., Baines, I., Burton, M. G. 2011, MNRAS, in press arXiv:1103.3910

Ossenkopf, V., \& Henning, T. 1994, A\&A, 291, 943

Pérault, M. et al. 1996, A\&A, 315, L165

Peretto, N. \& Fuller, G. A. 2010, ApJ, 723, 555

Pillai, T., Wyrowski, F., Carey, S. J., \& Menten 2006, A\&A, 450, 569

Ragan S. E., Bergin, E. A., and Gutermuth, R. A., 2009, ApJ, 698, 324

Rathborne, J. M., Jackson, J. M., \& Simon, R. 2006, ApJ, 641, 389

Tan, J. C. 2000, ApJ, 536, 173

Tan, J. C. 2007, in Triggered Star Formation in a Turbulent ISM, eds. B. G. Elmegreen \& J. Palous, IAUS 237, CUP, p258

Teyssier, D., Hennebell, P., Pérault, M., 2002, A\&A, 382, 624

Thomas, H. S., Fuller, G. A., 2008, A\&A, 479, 751T

Tielens, A. G. G. M., \& Allamandola, L. J. 1987, in Interstellar Processes, ed. D. J. Hollenbach \& H. A. Thronson, Jr. (Dordrecht: Kluwer), 397

Visser, R., van Dishoeck, E. F., \& Black, J. H., 2009, A\&A, 503, 323

Weingartner, J. C., \& Draine, B. T. 2001, ApJ, 548, 296

Whittet, D. C. B, Goldsmith, P. F., \& Pineda, J. L. 2010, ApJ, 720, 259

Willacy, K., Langer, W. D., Velusamy, T., 1998, ApJ, 507L, 171W

Wilson, T. L., \& Rood, R. T., 1994, ARA\&A, 32, 191

Zhang, Q., Wang, Y., Pillai, T., Rathborne, J., 2008, ApJ, 696, $268 Z$ 

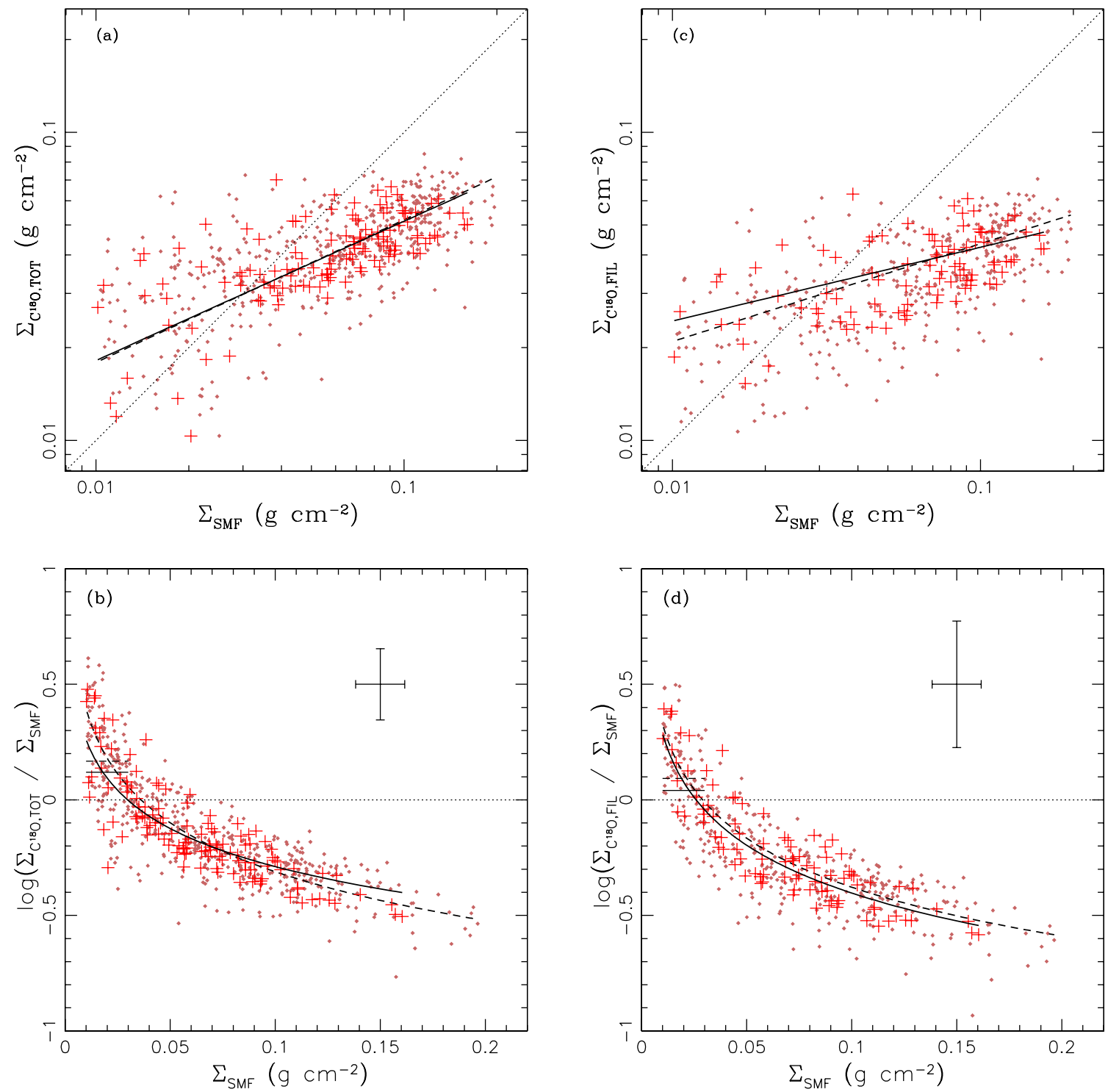

Fig. 3.- Evidence for CO depletion. (a) Top Left: Comparison of $\Sigma_{\mathrm{C}^{18} \mathrm{O} \text {, тот }}$ (i.e. Case 1) and $\Sigma_{\mathrm{SMF}}$ for all $\mathrm{C}^{18} \mathrm{O}(1-0)(\mathrm{crosses})$ and $\mathrm{C}^{18} \mathrm{O}(2-1)$ (dots) pixels for which both $\Sigma_{\mathrm{C} 18 \mathrm{O}}$ and $\Sigma_{\mathrm{SMF}}>0.01 \mathrm{~g} \mathrm{~cm}^{-2}$ and the pixel is $<20 \%$ affected by $\Sigma_{\mathrm{SMF}}$ artifacts, e.g. due to MIR bright sources. The dotted line shows the condition $\Sigma_{\mathrm{C}^{18} \mathrm{O} \text {, TOT }}=\Sigma_{\mathrm{SMF}}$. The solid, dashed lines show the best-fit power law relations to the $\mathrm{C}^{18} \mathrm{O}(1-0), \mathrm{C}^{18} \mathrm{O}(2-1)$ resolution data, respectively. (b) Bottom Left: Ratio $\Sigma_{\mathrm{C}^{18} \mathrm{O}, \mathrm{TOT}} / \Sigma_{\mathrm{SMF}}$ (i.e. Case 1 ) versus $\Sigma_{\mathrm{SMF}}$, with the same symbol and line notation as in (a). The horizontal solid, dashed lines from $0.01<\Sigma_{\mathrm{SMF}} / \mathrm{g} \mathrm{cm}^{-2}<0.03$ indicate the mean values of the data in this range for the $\mathrm{C}^{18} \mathrm{O}(1-0), \mathrm{C}^{18} \mathrm{O}(2-1)$ resolution data, respectively. The cross in the upper-right corner indicates typical estimated uncertainties. (c) Top Right: Same as (a), but now estimating $\Sigma_{\mathrm{C} 18} \mathrm{O}_{\mathrm{FIL}}$ from molecular gas associated with the filament after envelope subtraction (Case 2) in strips 2, 3 and 4. (d) Bottom Right: Same as (b), but for Case 2 . Both (b) and (d) show that $\Sigma_{\mathrm{C} 18 \mathrm{O}} / \Sigma_{\mathrm{SMF}}$ decreases by up to a factor of $\sim 5$ as $\Sigma_{\mathrm{SMF}}$ increases from $\sim 0.02 \mathrm{~g} \mathrm{~cm}^{-2}$ up to $\sim 0.2 \mathrm{~g} \mathrm{~cm}^{-2}$. 